\title{
Fixed-Point Posets in Theories of Truth
}

\author{
Stephen Mackereth
}

Received: date / Accepted: date

\begin{abstract}
We show that any coherent complete partial order (ccpo) is obtainable as the fixed-point poset of the strong Kleene jump of a suitably chosen first-order ground model. This is a strengthening of Visser's result that any finite ccpo is obtainable in this way. The same is true for the van Fraassen supervaluation jump, but not for the weak Kleene jump.
\end{abstract}

Keywords Truth $\cdot$ Paradox $\cdot$ Fixed-point theories $\cdot$ Coherent complete partial order

\section{Introduction}

Suppose we have a model of a first-order language, such that the domain of the model contains the sentences of that very language. The predicates of the language are interpreted as being applicable to the sentences of that very language. Can such a model contain its own truth-predicate? In a classical setting, the answer is "Not always." The liar paradox threatens.

But suppose that we allow three truth-values: t,f, and $\mathbf{n}$, or True, False, and Neither. Kripke [2] and Martin \& Woodruff [3] independently showed how a truthpredicate may be added to any three-valued model of any first-order language. To this end, they each defined a jump operation $\rho_{M}$ relative to a model $M$ and a threevalued logic $\rho .{ }^{1}$ The jump operation maps a possible interpretation $g \in\{\mathbf{t}, \mathbf{f}, \mathbf{n}\}^{D}$ of a designated predicate symbol $T$ to the function capturing the semantic values of the sentences of the language if $T$ were interpreted by $g$. Any viable candidate for the interpretation of the truth-predicate ought to be a fixed point of the jump operation. Kripke and Martin \& Woodruff showed that, in this three-valued setting, their jump

S. Mackereth

University of Pittsburgh, Pittsburgh, PA 15260, USA

E-mail: sgm32@pitt.edu

1 Kripke used the strong Kleene scheme $\kappa$ as his three-valued logic, while Martin \& Woodruff used the weak Kleene scheme $\mu$. These schemes, and also the van Fraassen supervaluation scheme $\sigma$, will be described in detail in the next section. 
operations always have at least one fixed point. In fact there is always a least fixed point, if we partially order possible interpretations of $T$ by "determinateness" (thinking of $\mathbf{n}$ as a less determinate truth-value than $\mathbf{t}, \mathbf{f}){ }^{2}$

Visser [6] inquired into the structure of the posets of fixed points of jump operations. He proved that the fixed-point poset of any monotone jump operation is a coherent complete partial order, to be defined in the next section ([6], 186, Lemma $15)$. Visser further observed that, for any finite ccpo $\mathscr{X}$, there is a ground model $M$ such that the fixed-point poset for the strong Kleene jump $\kappa_{M}$ is isomorphic to $\mathscr{X}$ ([6], 193, Remark 33). In fact, for finite ccpos, such a model can be constructed for a language containing only sentential connectives and constants.

This paper proves two main theorems. Theorem 1 generalizes Visser's observation: we show that for any ccpo $\mathscr{X}$, there is a ground model $M$ such that the fixedpoint poset for $\kappa_{M}$ is isomorphic to $\mathscr{X}$. The same holds if $\kappa_{M}$ is replaced by the van Fraassen supervaluation jump, $\sigma_{M}$. Theorem 2 shows that the analogous claim does not hold for the weak Kleene jump, $\mu_{M}$. Indeed, we exhibit a five-point ccpo $\mathscr{X}$ for which there is no ground model $M$ such that the fixed-point poset for $\mu_{M}$ is isomorphic to $\mathscr{X}$.

\section{Preliminary definitions}

\subsection{Definitions concerning truth}

Let $L$ be a first-order language. Let $L^{+}$be the language obtained by adding a oneplace predicate symbol $T$ to $L$, and let $\operatorname{Sent}\left(L^{+}\right)$denote the set of sentences of $L^{+}$.

Suppose further that we are given a three-valued model $M=(D, I)$ for $L$, such that $\operatorname{Sent}\left(L^{+}\right) \subseteq D$. The interpretation function $I$ interprets all the constant symbols, function symbols, and predicate symbols of $L$. Each $n$-place predicate symbol is assigned as its interpretation an element of $\{\mathbf{t}, \mathbf{f}, \mathbf{n}\}{ }^{D^{n}}$, where $\mathbf{t}, \mathbf{f}, \mathbf{n}$ are the three truth-values: True, False, and Neither. We shall call $M$ the ground model.

There are various three-valued schemes $\rho$ available for interpreting the sentential connectives and the quantifiers. We shall consider the strong Kleene $(\kappa)$, weak Kleene $(\mu)$, and van Fraassen supervaluation $(\sigma)$ schemes.

In the strong Kleene scheme, a conjunction $\varphi \& \psi$ is true if both conjuncts are true; false, if at least one conjunct is false; neither, otherwise. A negation $\neg \varphi$ is true if $\varphi$ is false; false if $\varphi$ is true; neither if $\varphi$ is neither. We also define the notion $\forall Z$, where $Z$ is a set of truth-values, as the generalized conjunction of all the truth-values in $Z$. Thus $\forall Z$ is true if $Z=\{\mathbf{t}\}$; false, if $\mathbf{f} \in Z$; neither, otherwise. The other logical constants may be defined in terms of these in the usual way.

In the weak Kleene scheme, a conjunction $\varphi \& \psi$ is true if both conjuncts are true; neither, if at least one conjunct is neither; false, otherwise. Negation is the same as in the strong Kleene scheme. The notion $\forall Z$ generalizes the weak Kleene conjunction: so, $\forall Z$ is true if $Z=\{\mathbf{t}\}$; neither, if $\mathbf{n} \in Z$; false, otherwise.

\footnotetext{
2 For a characterization of many-valued logical schemes whose corresponding jump operations are guaranteed to have fixed points, see [4], [5].
} 
The supervaluation scheme is not truth-functional. In the supervaluation scheme, a sentence $\varphi$ is true (false) in a three-valued model $M=(D, I)$ iff it is true (false) in all classical models $M^{\prime}=\left(D, I^{\prime}\right)$ that meet the following two conditions:

(i) if $X$ is a non-predicate symbol, then $I^{\prime}(X)=I(X)$,

(ii) for each predicate symbol $R$, for all $d \in D$, if $I(R)(d) \in\{\mathbf{t}, \mathbf{f}\}$, then $I^{\prime}(R)(d)=$ $I(R)(d)$

The data can be summarized as $\mathscr{L}=(L, M, \rho)$.

Suppose we are given $\mathscr{L}$ as above, and $\rho \in\{\kappa, \mu\}$. Let $s$ be a variable assignment function for $\mathscr{L}$, i.e., a function mapping the variables of $L$ into $D$. Define the semantic value function $\mathrm{Val}_{\mathscr{L}, s}$ inductively, as in Gupta \& Belnap ([1], 2A.4). Suppose that $c$ is a constant symbol, $x$ is a variable symbol, $t_{1}, \cdots, t_{n}$ are terms, $f$ is an $n$-ary function symbol, $R$ is an $n$-ary predicate symbol, and $\varphi, \psi$ are formulas. Then:

$$
\begin{aligned}
\operatorname{Val}_{\mathscr{L}, s}(c) & =I(c) \\
\operatorname{Val}_{\mathscr{L}, s}(x) & =s(x) \\
\operatorname{Val}_{\mathscr{L}, s}\left(f\left(t_{1}, \cdots, t_{n}\right)\right) & =I(f)\left(\operatorname{Val}_{\mathscr{L}, s}\left(t_{1}\right), \cdots, \operatorname{Val}_{\mathscr{L}, s}\left(t_{n}\right)\right) \\
\operatorname{Val}_{\mathscr{L}, s}\left(R\left(t_{1}, \cdots, t_{n}\right)\right) & =I(R)\left(\operatorname{Val}_{\mathscr{L}, s}\left(t_{1}\right), \cdots, \operatorname{Val}_{\mathscr{L}, s}\left(t_{n}\right)\right) \\
\operatorname{Val}_{\mathscr{L}, s}(\neg \varphi) & =\neg \operatorname{Val}_{\mathscr{L}, s}(\varphi) \\
\operatorname{Val}_{\mathscr{L}, s}(\varphi \& \psi) & =\operatorname{Val}_{\mathscr{L}, s}(\varphi) \& \operatorname{Val}_{\mathscr{L}, s}(\psi) \\
\operatorname{Val}_{\mathscr{L}, s}(\forall x \varphi) & =\forall\left\{\operatorname{Val}_{\mathscr{L}, s[d / x]}(\varphi): d \in D\right\} .
\end{aligned}
$$

There is a slight abuse of notation here. On the left-hand side, we are treating the connective and quantifier symbols as mere symbols of the language $L$, while on the right-hand side, we are treating those symbols as interpreted according to the scheme $\rho$.

The semantic value of a sentence is independent of the choice of $s$, which justifies the notation $\operatorname{Val}_{\mathscr{L}}(\varphi)$ when $\varphi$ is a sentence. We also use this notation when $\rho=\sigma$.

Let $g \in\{\mathbf{t}, \mathbf{f}, \mathbf{n}\}^{D}$. Let $M+g=\left(D, I^{\prime}\right)$ denote the model of $L^{+}$in which $I^{\prime}(T)=g$ and $I^{\prime}=I$ otherwise. We write $\mathscr{L}+g=\left(L^{+}, M+g, \rho\right)$.

We now define the jump function $\rho_{M}:\{\mathbf{t}, \mathbf{f}, \mathbf{n}\}^{D} \rightarrow\{\mathbf{t}, \mathbf{f}, \mathbf{n}\}^{D}$ for the scheme $\rho$ and the ground model $M$, as follows:

$$
\rho_{M}(g)(d)= \begin{cases}\operatorname{Val}_{\mathscr{L}+g}(d) & \text { if } d \in \operatorname{Sent}\left(L^{+}\right) \\ \mathbf{f} & \text { otherwise. }\end{cases}
$$

The jump function tells us which sentences of $L^{+}$are true if $T$ is interpreted as $g$ and everything else is interpreted as in $M$.

\subsection{Definitions concerning ccpos}

Let $\mathscr{X}=(X, \leq)$ be any poset. We say that a set $Y \subseteq X$ is consistent if every pair $\{x, y\} \subseteq Y$ has an upper bound in $\mathscr{X}$, i.e., for any such pair there exists $z \in X$ such that $x \leq z$ and $y \leq z$. $^{3}$

\footnotetext{
3 Not to be confused with the more common notion of a directed set. Recall that a set $Y \subseteq X$ is directed if $Y \neq \varnothing$ and every pair $\{x, y\} \subseteq Y$ has an upper bound in $Y$. So, every directed set is consistent, but not vice versa.
} 
Define a coherent complete partial order (ccpo) to be a poset in which every consistent subset $Y \subseteq X$ has a least upper bound in $\mathscr{X} .{ }^{4}$ We denote this least upper bound by $\sup _{\mathscr{X}} Y$, or just $\sup Y$.

The set $\{\mathbf{t}, \mathbf{f}, \mathbf{n}\}^{D}$ has a natural partial ordering $\preccurlyeq$, defined as follows. If $f, g \in$ $\{\mathbf{t}, \mathbf{f}, \mathbf{n}\}^{D}$, let:

$$
f \preccurlyeq g \Longleftrightarrow(\forall d \in D)(f(d) \in\{\mathbf{t}, \mathbf{f}\} \Longrightarrow f(d)=g(d)) .
$$

This is motivated by thinking of $\{\mathbf{t}, \mathbf{f}, \mathbf{n}\}$ as partially ordered by "determinateness": $\mathbf{n}$ is a less determinate truth-value than $\mathbf{t}$,f. Then $\preccurlyeq$ is the induced pointwise order on the set of functions $\{\mathbf{t}, \mathbf{f}, \mathbf{n}\}^{D}$.

We note that $\rho_{M}(\rho=\kappa, \mu, \sigma)$ is a monotone function on $\left(\{\mathbf{t}, \mathbf{f}, \mathbf{n}\}^{D}, \preccurlyeq\right)$.

Let $F \subseteq\{\mathbf{t}, \mathbf{f}, \mathbf{n}\}^{D}$ be the set of all fixed points of $\rho_{M}$. We call $\mathscr{F}=(F, \preccurlyeq)$ the fixed-point poset for $\rho_{M}$, where the ordering $\preccurlyeq$ is inherited from $\{\mathbf{t}, \mathbf{f}, \mathbf{n}\}^{D}$.

As noted above, Visser proved that the fixed-point poset for any monotone jump function is a ccpo ([6], 186, Lemma 15).

\section{Fixed-point posets for the strong Kleene jump}

Theorem 1 For any ccpo $\mathscr{X}=(X, \leq)$, there exists a ground model $M$ such that the fixed-point poset of the strong Kleene jump $\kappa_{M}$ is isomorphic to $\mathscr{X}$.

Proof Let the least member of $X$ be denoted by 0 . We will use $x, y, z$ to denote arbitrary members of $X$, and $\Gamma$ to denote an arbitrary subset of $X$.

Let $L$ contain variable symbols $k, m, n$, binary predicate symbols $R, S$, and $\in$, and constant symbols $a_{x}$ (one for each $x \in X-\{0\}$ ) and $b_{x}$ (one for each $\left.x \in X\right){ }^{5}$

We will specify the model momentarily. Then, in Lemmas 1 and 2, we construct for each $z \in X$ a corresponding fixed point $\tilde{g}_{z}$. These fixed points are all distinct. In Lemma 3, we show that there are no other fixed points. Finally, we show that the fixed points of $\kappa_{M}$ are ordered isomorphically to $\mathscr{X}$.

Specification of the model $M=(D, I)$. First, some auxiliary definitions. Let the formulas $\alpha_{x}, \beta_{x}, \gamma_{x}, \delta_{x}$ for each $x \in X-\{0\}$ be defined as follows:

$$
\begin{aligned}
\beta_{x} & =\exists k\left(R a_{x} k \& T k\right) \\
\gamma_{x} & =\exists n\left(S n a_{x} \& \forall k(k \in n \rightarrow T k)\right) \\
\delta_{x} & =\forall k\left(\left[R k k \& \neg \exists m\left(R a_{x} m \& R k m\right)\right] \rightarrow \neg T k\right) \\
\alpha_{x} & =\left(T a_{x} \vee \neg T a_{x} \vee \beta_{x} \vee \gamma_{x}\right) \& \delta_{x} .
\end{aligned}
$$

Let $A=\left\{\alpha_{x}: x \in X-\{0\}\right\} \subseteq \operatorname{Sent}\left(L^{+}\right)$.

Let $D=\operatorname{Sent}\left(L^{+}\right) \cup \mathscr{P}(A)$.

${ }^{4}$ Again, not to be confused with directed-complete partial orders (dcpos). Every ccpo is a pointed dcpo, but not vice versa. For example, let $X=\mathscr{P}(\{1,2,3\})-\{\{1,2,3\}\}$. Then $\mathscr{X}=(X, \subseteq)$ is pointed directedcomplete, but not coherent complete. Indeed, the set $Y=\{\{1\},\{2\},\{3\}\} \subseteq X$ is consistent, but has no least upper bound in $\mathscr{X}$.

5 If $X$ is countable, we do not need so many constants $b_{x}$. It suffices to take a single constant $b_{0}$. 
Let $I\left(a_{x}\right)=\alpha_{x}$.

Let $I\left(b_{x}\right)=\neg T b_{x}$.

We assign $R$ a classical extension corresponding to the order relation of $\mathscr{X}$. Let $I(R)\left(d_{1}, d_{2}\right)=\mathbf{t}$ just in case $d_{1}=\alpha_{x}$ and $d_{2}=\alpha_{y}$ such that $x \leq y$. Otherwise, $I(R)\left(d_{1}, d_{2}\right)=\mathbf{f}$.

We assign $S$ a classical extension as follows: $I(S)\left(d_{1}, d_{2}\right)=\mathbf{t}$ just in case $d_{1} \in$ $\mathscr{P}(A)$ and $d_{2}=\alpha_{x} \in A$ such that $x=\sup _{\mathscr{X}}\left\{y \in X: \alpha_{y} \in d_{1}\right\}$. Otherwise, $I(S)\left(d_{1}, d_{2}\right)=$ f.

We assign $\in$ the usual interpretation: $I(\in)\left(d_{1}, d_{2}\right)=\mathbf{t}$ just in case $d_{2} \in \mathscr{P}(A)$ and $d_{1} \in d_{2}$. Otherwise, $I(\in)\left(d_{1}, d_{2}\right)=\mathbf{f}$.

We are now able to motivate our choice of $I\left(a_{x}\right)$. Intuitively, the $\beta_{x}$ disjunct ensures that, if any sentence $\alpha_{y}$ with $y \geq x$ is true, then $\alpha_{x}$ is true as well. The $\gamma_{x}$ disjunct ensures that, if $x$ is the supremum of some set $\Gamma \subseteq \mathscr{X}$, and $\alpha_{y}$ is true for all $y \in \Gamma$, then $\alpha_{x}$ is true as well. The $\delta_{x}$ conjunct ensures that, if any sentence $\alpha_{y}$ such that $\{x, y\}$ has no upper bound in $\mathscr{X}$ is true, then $\alpha_{x}$ is false.

More definitions. Our first task will be to write down some fixed points of $\kappa_{M}$.

Say that $g \in\{\mathbf{t}, \mathbf{f}, \mathbf{n}\}^{D}$ is a precursor function if $\kappa_{M}(g)(d)=g(d)$ for all $d \in A$, and $g(d)=\mathbf{n}$ if $d \in D-A$.

The definition of "precursor function" is motivated by the following observations. If $g$ is a precursor function, then $\kappa_{M}(g) \succcurlyeq g$. So, as Kripke notes, if the jump is iterated transfinitely, one eventually obtains a fixed point $\tilde{g}=\kappa_{M}{ }^{\infty}(g)$ ([2], 704). Moreover, $\left(\kappa_{M}(g)\lceil A)=(g\lceil A)\right.$, and hence $(\tilde{g}\lceil A)=(g\lceil A)$. This property will be important later.

In Lemma 1, we will specify some functions $g_{z}$ (one for each $z \in X$ ) and prove that they are precursor functions. In Lemma 2, we will show that for each $z \in X$, there can be at most one fixed point $h$ of $\kappa_{M}$ such that $\left(h\lceil A)=\left(g_{z}\lceil A)\right.\right.$.

Lemma 1 The following are all precursor functions.

(i) The function $g_{0} \in\{\boldsymbol{t}, \boldsymbol{f}, \boldsymbol{n}\}^{D}$ such that $g_{0}(d)=\boldsymbol{n}$ for all $d \in D$.

(ii) For each $z \in X-\{0\}$, the function $g_{z} \in\{\boldsymbol{t}, \boldsymbol{f}, \boldsymbol{n}\}^{D}$ such that $g_{z}(d)=\boldsymbol{n}$ if $d \in$ $D-A$, and:

$$
g_{z}: \alpha_{y} \mapsto \begin{cases}\boldsymbol{t} & \text { if } y \leq z \\ \boldsymbol{f} & \text { if }\{y, z\} \text { has no upper bound in } \mathscr{X} \\ \boldsymbol{n} & \text { otherwise. }\end{cases}
$$

Proof It is easy to show that $g_{0}$ is a precursor function. We omit the proof. Now consider $g_{z}$ with $z \in X-\{0\}$. We need to check that $\kappa_{M}\left(g_{z}\right)\left(\alpha_{x}\right)=\operatorname{Val}_{\mathscr{L}+g_{z}}\left(\alpha_{x}\right)=$ $g_{z}\left(\alpha_{x}\right)$ for all $x \in X-\{0\}$. Let us calculate $\operatorname{Val}_{\mathscr{L}+g_{z}}\left(\alpha_{x}\right)$. We divide into three cases, corresponding to the cases in the definition of $g_{z}$.

Case 1: $x \leq z$. In this case, we have $\operatorname{Val}_{\mathscr{L}+g_{z}}\left(T a_{x}\right)=g_{z}\left(\alpha_{x}\right)=\mathbf{t}$ by the definition of $g_{z}$. So, $\operatorname{Val}_{\mathscr{L}+g_{z}}\left(T a_{x} \vee \neg T a_{x} \vee \beta_{x} \vee \gamma_{x}\right)=\mathbf{t}$. We must show that $\operatorname{Val}_{\mathscr{L}+g_{z}}\left(\delta_{x}\right)=\mathbf{t}$ also. Indeed, let $s$ be an arbitrary variable assignment. If $s(k) \notin A$, then $\operatorname{Val}_{\mathscr{L}+g_{z}, s}(R k k)=$ f, so $\operatorname{Val}_{\mathscr{L}+g_{z}, s}\left(\left[R k k \& \neg \exists m\left(R a_{x} m \& R k m\right)\right] \rightarrow \neg T k\right)=\mathbf{t}$. 
If $s(k) \in A$, then $\operatorname{Val}_{\mathscr{L}+g_{z}, s}\left(\neg \exists m\left(R a_{x} m \& R k m\right)\right)=\mathbf{t}$ just in case $s(k)=\alpha_{y}$ for some $y$ such that $\{x, y\}$ has no upper bound. But for such $y$, there is no upper bound for $\{z, y\}$ either, since $x \leq z$. So, for all such $y$, we have $\operatorname{Val}_{\mathscr{L}+g_{z}, s}(T k)=g_{z}\left(\alpha_{y}\right)=\mathbf{f}$, and hence $\operatorname{Val}_{\mathscr{L}+g_{z}, s}\left(\left[R k k \& \neg \exists m\left(R a_{x} m \& R k m\right)\right] \rightarrow \neg T k\right)=\mathbf{t}$.

Hence, $\kappa_{M}\left(g_{z}\right)\left(\alpha_{x}\right)=g_{z}\left(\alpha_{x}\right)=\mathbf{t}$.

Case 2: $\{x, z\}$ has no upper bound. Since $z \leq z$, the definition of $g_{z}$ entails that $g_{z}\left(\alpha_{z}\right)=\mathbf{t}$. So, when $s(k)=\alpha_{z}$, we have $\operatorname{Val}_{\mathscr{L}+g_{z}, s}\left(\left[R k k \& \neg \exists m\left(R a_{x} m \& R k m\right)\right] \rightarrow\right.$ $\neg T k)=\mathbf{f}$.

Hence, $\kappa_{M}\left(g_{z}\right)\left(\alpha_{x}\right)=g_{z}\left(\alpha_{x}\right)=\mathbf{f}$.

Case 3: $x \not \leq z$, but there is an upper bound for $\{x, z\}$. In this case, we have $\operatorname{Val}_{\mathscr{L}+g_{z}}\left(T a_{x}\right)=$ $\operatorname{Val}_{\mathscr{L}+g_{z}}\left(\neg T a_{x}\right)=\mathbf{n}$.

Moreover, $\operatorname{Val}_{\mathscr{L}+g_{z}}\left(\beta_{x}\right) \neq \mathbf{t}$. For if $s(k)=\alpha$ such that $y \geq x$, then $y \not \leq z$, so $\operatorname{Val}_{\mathscr{L}+g_{z}, s}(T k) \neq \mathbf{t}$.

Moreover, $\operatorname{Val}_{\mathscr{L}+g_{z}}\left(\gamma_{x}\right) \neq \mathbf{t}$. For suppose that $s(n) \in \mathscr{P}(A)$. Let $\Gamma=\left\{y \in X: \alpha_{y} \in\right.$ $s(n)\}$, and suppose further that $g_{z}\left(\alpha_{y}\right)=\mathbf{t}$ for all $y \in \Gamma$. Then $z$ is an upper bound of $\Gamma$, and hence $\sup \Gamma \leq z$. But $x \not z z$. So $\sup \Gamma \neq x$.

Finally, $\operatorname{Val}_{\mathscr{L}+g_{z}}\left(\delta_{x}\right) \neq \mathbf{f}$. For suppose that $s(k)=\alpha_{y}$ such that $\{y, x\}$ has no upper bound. Then $y \not z z$, so $\operatorname{Val}_{\mathscr{L}+g_{z}, s}(T k) \neq \mathbf{t}$. Hence $\operatorname{Val}_{\mathscr{L}+g_{z}, s}(\neg T k) \neq \mathbf{f}$.

Hence, $\kappa_{M}\left(g_{z}\right)\left(\alpha_{x}\right)=g_{z}\left(\alpha_{x}\right)=\mathbf{n}$.

So, $g_{z}$ is a precursor function.

Lemma 2 If $h_{1}, h_{2}$ are fixed points of $\kappa_{M}$, and $\left(h_{1}\lceil A)=\left(h_{2}\lceil A)\right.\right.$, then $h_{1}=h_{2}$.

Proof Our proof is based on Gupta \& Belnap's proof of their Main Lemma ([1], 6A.4). It suffices to show that $M+h_{1}, M+h_{2}$ are isomorphic $L^{+}$-structures. For in that case, the same sentences will be true in each model. So we will have $h_{1}=$ $\kappa_{M}\left(h_{1}\right)=\kappa_{M}\left(h_{2}\right)=h_{2}$.

We construct the isomorphism $\Psi: D \rightarrow D$ as follows.

Let $Y=\operatorname{Sent}\left(L^{+}\right)-\left(A \cup\left\{\neg T b_{x}: x \in X\right\}\right)$. Let $\Psi$ be identity on $D-Y$.

Following Gupta $\&$ Belnap ([1], 2D.2), we say that an $m$-ary function $f$ with domain $D$ is $Y$-neutral just in case, for any $1 \leq i \leq m$ and any $d_{i}, d_{i}^{\prime} \in Y$ :

$$
f\left(d_{1}, \cdots, d_{i}, \cdots, d_{m}\right)=f\left(d_{1}, \cdots, d_{i}^{\prime}, \cdots, d_{m}\right) .
$$

Intuitively, a function is $Y$-neutral if it does not make distinctions between members of $Y$.

Observe that $I(R), I(S), I(\in)$ are all $Y$-neutral. Also, note that for any name $a_{x}$ or $b_{x}$, we have $I\left(a_{x}\right) \notin Y, I\left(b_{x}\right) \notin Y$.

Define the sets $Y_{\mathbf{v}}^{i}$ for $i \in\{1,2\}$ and $\mathbf{v} \in\{\mathbf{t}, \mathbf{f}, \mathbf{n}\}$ by $Y_{\mathbf{v}}^{i}=\left\{\varphi \in Y: h_{i}(\varphi)=\mathbf{v}\right\}$. We claim that each of these six sets has cardinality equal to $\max \left\{\boldsymbol{\aleph}_{0},|X|\right\}$. Indeed, note that:

$$
\begin{aligned}
h_{1}\left(R a_{x} a_{x}\right)=h_{2}\left(R a_{x} a_{x}\right) & =\mathbf{t} \\
h_{1}\left(\neg R a_{x} a_{x}\right)=h_{2}\left(\neg R a_{x} a_{x}\right) & =\mathbf{f} \\
h_{1}\left(T b_{x}\right)=h_{2}\left(T b_{x}\right) & =\mathbf{n},
\end{aligned}
$$


and $h_{i}(\varphi \& \varphi)=h_{i}(\varphi)$ for any sentence $\varphi$. So each $Y_{\mathbf{v}}^{i}$ is at least countably infinite, and $\left|Y_{\mathbf{v}}^{i}\right|=|X|$ when $|X|$ is infinite.

We can now complete the construction of $\Psi$. For each $\mathbf{v} \in\{\mathbf{t}, \mathbf{f}, \mathbf{n}\}$, let $f_{\mathbf{v}}: Y_{\mathbf{v}}^{1} \rightarrow Y_{\mathbf{v}}^{2}$ be a bijection. Then let $\Psi$ be equal to $f_{\mathbf{v}}$ on $Y_{\mathbf{v}}^{1}$, for each $\mathbf{v} \in\{\mathbf{t}, \mathbf{f}, \mathbf{n}\}$.

By definition, $\Psi$ preserves the interpretation of $T$. Also, $\Psi$ preserves the interpretation of everything else, since everything else is $Y$-neutral. So $\Psi$ is an isomorphism.

Recall that $\tilde{g}_{z}=\kappa_{M}^{\infty}\left(g_{z}\right)$ denotes the Kripke least fixed point obtained when $g_{z}$ is the initial interpretation of $T$. We observed earlier (when we defined "precursor function") that $\left(\tilde{g}_{z}\lceil A)=\left(g_{z}\lceil A)\right.\right.$.

Corollary 1 For each $z \in X$, there is exactly one fixed point that agrees with $g_{z}$ on $A$, namely $\tilde{g}_{z}$.

Lemma 3 If $h$ is a fixed point of $\kappa_{M}$, then $\left(h\lceil A)=\left(g_{z}\lceil A)\right.\right.$ for some $z \in X$.

Proof Suppose that $h$ is a fixed point of $\kappa_{M}$. Let $B=\left\{y \in X: h\left(\alpha_{y}\right)=\mathbf{t}\right\}$.

First, consider the case where $B=\varnothing$. Let $x \in X$ be arbitrary. Notice that, because $\alpha_{x}$ contains the $\neg T a_{x}$ disjunct, we can have $h\left(\alpha_{x}\right)=\mathbf{f}$ only if $\delta_{x}$ is false. But that can only happen if for some $y$, we have $h\left(\alpha_{y}\right)=\mathbf{t}$. But we supposed that $B=\varnothing$. Hence, $h\left(\alpha_{x}\right)=\mathbf{n}$ for all $x \in X$. So, $\left(h\lceil A)=\left(g_{0}\lceil A)\right.\right.$.

Now consider the case where $B \neq \varnothing$. We show that $B$ is closed downward and closed under sups (when they exist).

(i) Closed downward Suppose that $h\left(\alpha_{y}\right)=\mathbf{t}$, and let $x \leq y$. Since $h\left(\alpha_{y}\right)=\mathbf{t}$, we have that $\beta_{x}$ is true. Suppose for sake of contradiction that $h\left(\alpha_{x}\right) \neq \mathbf{t}$. Then $\delta_{x}$ must not be true, i.e., there must be some $w \in X$ such that $\{x, w\}$ has no upper bound and $h\left(\alpha_{w}\right) \neq \mathbf{f}$. But $x \leq y$, so $\{y, w\}$ has no upper bound either. Hence, $h\left(\alpha_{y}\right) \neq \mathbf{t}$, contradiction. So, $h\left(\alpha_{x}\right)=\mathbf{t}$.

(ii) Closed under sups Suppose that $h\left(\alpha_{y}\right)=\mathbf{t}$ for all $y \in \Gamma$, and $\sup \Gamma=x$. Then $\gamma_{x}$ is true. Suppose for a contradiction that $\delta_{x}$ is not true, i.e., there exists $w$ such that $\{x, w\}$ has no upper bound and $h\left(\alpha_{w}\right) \neq \mathbf{f}$. Now for each $y \in \Gamma$, since $h\left(\alpha_{y}\right)=\mathbf{t}$, it must be that $\{y, w\}$ has an upper bound. So, the set $\Gamma \cup\{w\}$ is consistent. Since $\mathscr{X}$ is a ccpo, it follows that $\sup (\Gamma \cup\{w\})$ exists and is an upper bound for $\{x, w\}$, contradiction. So, $h\left(\alpha_{x}\right)=\mathbf{t}$.

Next, we observe that $B$ is consistent. Indeed, suppose not. Then some pair $\{x, y\} \subseteq$ $B$ has no upper bound. But then $\delta_{x}$ is falsified. Hence, $h\left(\alpha_{x}\right)=\mathbf{f}$, contradicting that $x \in B$.

So, $B$ is consistent. Since $\mathscr{X}$ is a ccpo, $B$ has a least upper bound. Since $B$ is closed under sups, it follows that $B$ has a greatest element, call it $z$. Then, since $B$ is closed downward, we have that $B=\{y \in X: y \leq z\}$.

We now proceed to show that $\left(h\lceil A)=\left(g_{z}\lceil A)\right.\right.$. Divide into the three cases that feature in the definition of $g_{z}$.

Case 1: $x \leq z$. In this case, we have already shown that $h\left(\alpha_{x}\right)=g_{z}\left(\alpha_{x}\right)=\mathbf{t}$. 
Case 2: $\{x, z\}$ has no upper bound. Suppose that $h\left(\alpha_{x}\right) \neq \mathbf{f}$. Then $\delta_{z}$ is not true. So $h\left(\alpha_{z}\right) \neq \mathbf{t}$, contradiction.

Case 3: $x \not z z$, but there is an upper bound for $\{x, z\}$. Suppose for sake of contradiction that $h\left(\alpha_{x}\right) \neq \mathbf{n}$. By our characterization of $B, h\left(\alpha_{x}\right) \neq \mathbf{t}$. So $h\left(\alpha_{x}\right)=\mathbf{f}$. Since $\alpha_{x}$ contains the $\neg T a_{x}$ disjunct, this can only happen if $\delta_{x}$ is false, i.e., if there is some $y$ such that $\{x, y\}$ have no upper bound and $h\left(\alpha_{y}\right)=\mathbf{t}$.

But by our characterization of $B$, if $h\left(\alpha_{y}\right)=\mathbf{t}$, then $y \leq z$, which would imply that $\{y, x\}$ do have an upper bound. Contradiction.

This concludes the lemma.

Corollary 2 The set of fixed points of $\kappa_{M}$ is $\left\{\tilde{g}_{z}: z \in X\right\}$.

Let $F$ be the set of fixed points of $\kappa_{M}$, and let $\mathscr{F}=(F, \preccurlyeq)$. We will now show that $\mathscr{F}$ is order-isomorphic to $\mathscr{X}$. Specifically, we will show that the map $\mathscr{X} \rightarrow \mathscr{F}$ defined by $z \mapsto \tilde{g}_{z}$ is order-preserving. It suffices to show that, if $x<z$, then $\tilde{g}_{x} \prec \tilde{g}_{z}$.

Suppose that $x<z$. Let $\alpha_{y} \in A$ be arbitrary. We need only consider the cases where $g_{x}\left(\alpha_{y}\right) \neq \mathbf{n}$.

(i) If $y \leq x$, then $y \leq z$. So, if $g_{x}\left(\alpha_{y}\right)=\mathbf{t}$, then $g_{z}\left(\alpha_{y}\right)=\mathbf{t}$ too.

(ii) If $\{y, x\}$ has no upper bound, then $\{y, z\}$ has no upper bound either. So, if $g_{x}\left(\alpha_{y}\right)=\mathbf{f}$, then $g_{z}\left(\alpha_{y}\right)=\mathbf{f}$ too.

Hence, $g_{x} \preccurlyeq g_{z}$. Since $\kappa_{M}$ is monotonic, it follows that $\tilde{g}_{x} \preccurlyeq \tilde{g}_{z}$.

Now note that $\tilde{g}_{z}\left(a_{z}\right)=g_{z}\left(a_{z}\right)=\mathbf{t}$ but $\tilde{g}_{x}\left(a_{z}\right)=g_{x}\left(a_{z}\right) \neq \mathbf{t}$. Hence, $\tilde{g}_{x} \prec \tilde{g}_{z}$.

So, the map $z \mapsto \tilde{g}_{z}$ is order-preserving. Hence, $\mathscr{X}$ and $\mathscr{F}$ are order-isomorphic.

This completes the proof of Theorem 1 .

Remark 1 In the case where $X$ is finite, we can carry out the construction in a language containing only sentential connectives and constants. Let $L$ contain just the names $a_{x}$ for $x \in X-\{0\}$, and let $I$ be given by:

$I\left(a_{x}\right)=\left(T a_{x} \vee \neg T a_{x} \vee \bigvee_{y \geq x} T a_{y} \vee \bigvee_{\sup \left\{y_{1}, \cdots, y_{m}\right\}=x}\left(T a_{y_{1}} \& \cdots \& T a_{y_{m}}\right)\right) \& \bigwedge_{\{x, y\} \text { has no UB }} \neg T a_{y}$

Remark 2 In the case of the van Fraassen jump $\left(\sigma_{M}\right)$, essentially the same construction shows that, for any ccpo $\mathscr{X}=(X, \leq)$, there exists a ground model $M$ such that the fixed-point poset of $\sigma_{M}$ is isomorphic to $\mathscr{X}$. Take all the definitions as in Theorem 1 , except let the interpretation of the names $a_{x}(x \in X-\{0\})$ be given by:

$$
I\left(a_{x}\right)=\left(T a_{x} \vee T b_{0} \vee \beta_{x} \vee \gamma_{x}\right) \& \delta_{x},
$$

where $I\left(b_{0}\right)=\neg T b_{0}$. The proofs go through as before, except for some small modifications to Lemmas 1 and 3.

Remark 3 In the case of the weak Kleene jump $\left(\mu_{M}\right)$, a similar construction shows that for any tree $\mathscr{X}=(X, \leq)$, there exists a ground model $M$ such that the fixed-point poset of $\mu_{M}$ is isomorphic to $\mathscr{X}$. For the interpretations of the names $a_{x}$, we take:

$$
I\left(a_{x}\right)=\left(T a_{x} \vee \neg T a_{x}\right) \& \forall k\left(R k a_{x} \rightarrow T k\right) \& \delta_{x} .
$$




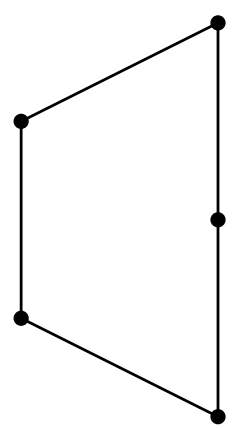

Fig. 1 The five-point non-distributive lattice $N_{5}$.

One can also obtain some non-trees; e.g., if $\mathscr{X}$ is the product poset of some trees, then there is a ground model $M$ such that fixed-point poset of $\mu_{M}$ is isomorphic to $\mathscr{X}$. However, not every poset $\mathscr{X}$ can be obtained, as we now show.

\section{Fixed-point posets for the weak Kleene jump}

Theorem 2 Let $\mathscr{X}=(X, \leq)$ be the five-point non-distributive lattice $N_{5}$ depicted in Fig. 1. Then there is no ground model $M=(D, I)$ such that the fixed-point poset of the weak Kleene jump $\mu_{M}$ is isomorphic to $\mathscr{X}$.

Proof Suppose that we are given $\mathscr{L}=(L, M, \mu)$. Let $F$ denote the set of fixed points of $\mu_{M}$, and let $\mathscr{F}=(F, \preccurlyeq)$.

Suppose for sake of contradiction that $\mathscr{F}$ is order-isomorphic to $\mathscr{X}$. Then there is a greatest fixed point $g_{\max }$. Note that if $g$ is any fixed point of $\mu_{M}$, then $g \preccurlyeq g_{\text {max }}$, so $g(\varphi) \in\left\{\mathbf{n}, g_{\max }(\varphi)\right\}$ for each $\varphi \in \operatorname{Sent}\left(L^{+}\right)$. We will sometimes write $\mathbf{v}_{\varphi}$ to represent the value of $g_{\max }(\varphi)$, when that value is not $\mathbf{n}$.

There is a least fixed point $g_{\text {min }}$. On one branch, there are two fixed points $g_{1}, g_{3}$ such that $g_{\text {min }} \prec g_{1} \prec g_{3} \prec g_{\text {max }}$. Since $g_{1} \prec g_{3}$, there is a sentence $\gamma$ such that $g_{3}(\gamma)=$ $g_{\text {max }}(\gamma) \neq \mathbf{n}$ but $g_{1}(\gamma)=\mathbf{n}$.

On the other branch, there is one more fixed point $g_{\min } \prec g_{2} \prec g_{\max }$. Note that $g_{2}$ is incomparable with each of $g_{1}, g_{3}$. Since $g_{1} \npreceq g_{2}$, there is a sentence $\alpha$ such that $g_{1}(\alpha)=g_{\max }(\alpha) \neq \mathbf{n}$ but $g_{2}(\alpha)=\mathbf{n}$. Since $g_{2} \npreceq g_{3}$, there is a sentence $\beta$ such that $g_{2}(\beta)=g_{\text {max }}(\beta) \neq \mathbf{n}$ but $g_{3}(\beta)=\mathbf{n}$. These relationships are depicted in Fig. 2 .

For future reference, let these constraints be denoted as follows:

$$
\begin{aligned}
\mathscr{C}_{1}(\alpha) & \Longleftrightarrow\left(g_{1}(\alpha) \neq \mathbf{n} \& g_{2}(\alpha)=\mathbf{n}\right) \\
\mathscr{C}_{2}(\beta) & \Longleftrightarrow\left(g_{2}(\beta) \neq \mathbf{n} \& g_{3}(\beta)=\mathbf{n}\right) \\
\mathscr{C}_{3}(\gamma) & \Longleftrightarrow\left(g_{3}(\gamma) \neq \mathbf{n} \& g_{1}(\gamma)=\mathbf{n}\right) \\
\mathscr{C}(\alpha, \beta, \gamma) & \Longleftrightarrow\left(\mathscr{C}_{1}(\alpha) \& \mathscr{C}_{2}(\beta) \& \mathscr{C}_{3}(\gamma)\right) .
\end{aligned}
$$

Here is the main idea of the proof. In Lemma 6, we will specify a transformation that takes a fixed point of $\mu_{M}$ and changes some of its values to $\mathbf{n}$ so as to yield 


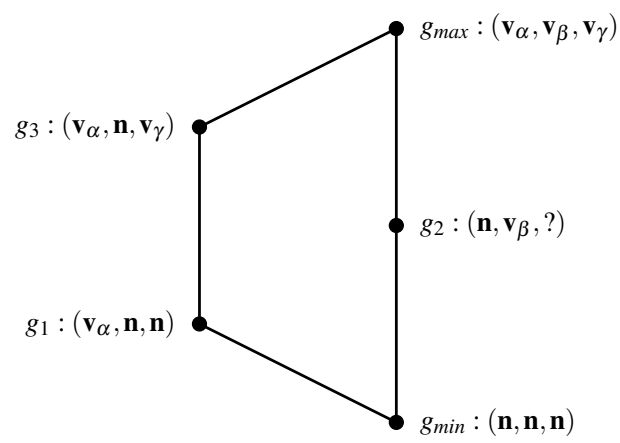

Fig. 2 The fixed points of $\mu_{M}$. For each fixed point $h$, the triple $(h(\alpha), h(\beta), h(\gamma))$ is displayed.

another fixed point of $\mu_{M}$. We will obtain a contradiction by showing that if $\mathscr{F}$ is isomorphic to $\mathscr{X}$, then $F$ is not closed under this transformation. Lemmas 4 and 5 provide ancillary technical support.

We begin by highlighting three key properties of the weak Kleene scheme, which we will use in the proofs of the lemmas.

Key properties of weak Kleene. Let $g \in\{\mathbf{t}, \mathbf{f}, \mathbf{n}\}^{D}$ be arbitrary, and let $s$ be an arbitrary assignment function. Let $k$ be a variable symbol. Then for any formulas $\psi, \theta$ of $L^{+}$:

$$
\begin{aligned}
\operatorname{Val}_{\mathscr{L}+g, s}(\psi \& \theta) & =\mathbf{n} \Longleftrightarrow\left(\operatorname{Val}_{\mathscr{L}+g, s}(\psi)=\mathbf{n} \vee \operatorname{Val}_{\mathscr{L}+g, s}(\theta)=\mathbf{n}\right) \\
\operatorname{Val}_{\mathscr{L}+g, s}(\neg \psi) & =\mathbf{n} \Longleftrightarrow \operatorname{Val}_{\mathscr{L}+g, s}(\psi)=\mathbf{n} \\
\operatorname{Val}_{\mathscr{L}+g, s}(\forall k \psi) & =\mathbf{n} \Longleftrightarrow \exists s^{\prime}\left(\operatorname{Val}_{\mathscr{L}+g, s^{\prime}}(\psi)=\mathbf{n}\right) .
\end{aligned}
$$

We will refer to these as the Conjunction Property, the Negation Property, and the Quantifier Property, respectively. If $\psi, \theta$ are sentences, then we can drop the assignment function $s$. For the Quantifier Property, we can also drop $s$ if $\forall k \psi$ is a sentence.

Definitions. For any formulas $\varphi, \psi$ of $L^{+}$, let $\operatorname{Subf}(\varphi, \psi)$ denote that $\varphi$ occurs as a subformula in $\psi$.

Say that a sentence $\varphi \in \operatorname{Sent}\left(L^{+}\right)$is basic if it has the form $T t$ for some closed term $t$, or the form $\forall k_{1} \cdots \forall k_{m} T t$, where $t=t\left(k_{1}, \cdots, k_{m}\right)$ is a term of $L^{+}$with exactly the free variables $k_{1}, \cdots, k_{m}$. In either case, say that $t$ is the principal term of $\varphi$, and $\varphi$ is the principal sentence for $t$. We use the notation $\forall \forall T t$ to denote the principal sentence for $t$.

Lemma 4 Let $\varphi$ be any formula of $L^{+}$. Let $f, h \in\{\boldsymbol{t}, \boldsymbol{f}, \boldsymbol{n}\}^{D}$ be such that:

1. there is an assignment function $s_{0}$ such that $\operatorname{Val}_{\mathscr{L}+f, s_{0}}(\varphi)=\boldsymbol{n}$,

2. for all assignment functions $s, \operatorname{Val}_{\mathscr{L}+h, s}(\varphi) \neq \boldsymbol{n}$.

Then there is a term $t$ such that:

a) $\operatorname{Subf}(T t, \varphi)$,

b) $\operatorname{Val}_{\mathscr{L}+f}(\forall \forall T t)=\boldsymbol{n}$ and $\operatorname{Val}_{\mathscr{L}+h}(\forall \forall T t) \neq \boldsymbol{n}$. 
Proof By induction on formulas $\varphi$. Assume as hypothesis of induction that for all subformulas of $\varphi$ satisfying (1) and (2), there is a term $t$ satisfying (a) and (b). We divide into three cases.

Case 1. Suppose $\varphi$ is an atomic formula. If $\varphi$ were an atomic formula of the ground language $L$, then $\operatorname{Val}_{\mathscr{L}+g, s}(\varphi)$ would not depend on $g$. But there is an $s_{0}$ such that $\operatorname{Val}_{\mathscr{L}+f, s_{0}}(\varphi) \neq \operatorname{Val}_{\mathscr{L}+h, s_{0}}(\varphi)$. So, $\varphi$ has the form $T t$ for some term $t$. By the Quantifier Property, the universal closure of $\varphi$ has the desired semantic values in $\mathscr{L}+f$, $\mathscr{L}+h$. Hence, we are done.

Case 2. Suppose $\varphi$ is of the form $\psi \& \theta$ or $\neg \psi$. Consider the first subcase, $\varphi=\psi \& \theta$. By hypothesis, there is an $s_{0}$ such that $\operatorname{Val}_{\mathscr{L}+f, s_{0}}(\varphi)=\mathbf{n}$. By the Conjunction Property, either $\operatorname{Val}_{\mathscr{L}+f, s_{0}}(\psi)=\mathbf{n}$ or $\operatorname{Val}_{\mathscr{L}+f, s_{0}}(\theta)=\mathbf{n}$. Moreover, since $\forall s\left(\operatorname{Val}_{\mathscr{L}+h, s}(\varphi) \neq\right.$ $\mathbf{n})$, it follows from the Conjunction Property that $\forall s\left(\operatorname{Val}_{\mathscr{L}+h, s}(\psi) \neq \mathbf{n}\right)$ and also $\forall s\left(\operatorname{Val}_{\mathscr{L}+h, s}(\boldsymbol{\theta}) \neq \mathbf{n}\right)$. Hence, we can apply the inductive hypothesis to one of $\psi, \theta$.

The other subcase, $\varphi=\neg \psi$, is very similar.

Case 3. Suppose now that $\varphi$ is of the form $\forall k \psi$. Recall that $\operatorname{Val}_{\mathscr{L}+f, s_{0}}(\varphi)=\mathbf{n}$. By the Quantifier Property, there is an assignment function $s^{\prime}$ such that $\operatorname{Val}_{\mathscr{L}+f, s^{\prime}}(\psi)=\mathbf{n}$. Also, since $\forall s\left(\operatorname{Val}_{\mathscr{L}+h, s}(\varphi) \neq \mathbf{n}\right)$, it follows that $\forall s\left(\operatorname{Val}_{\mathscr{L}+h, s}(\psi) \neq \mathbf{n}\right)$ too. Then apply the inductive hypothesis to $\psi$.

Corollary 3 If $\mathscr{F}$ is isomorphic to $\mathscr{X}$, then there exist basic sentences $\alpha, \beta, \gamma$ which satisfy the constraint $\mathscr{C}$.

Proof We noted earlier that, since $g_{1} \npreceq g_{2}$, there exists a sentence $\alpha$ satisfying $\mathscr{C}_{1}$. Choose some such $\alpha$. Then apply Lemma 4 with $\varphi=\alpha, f=g_{2}$, and $h=g_{1}$ to obtain a basic sentence $\forall \forall T t$ satisfying (a) and (b). Now, (b) says that $\forall \forall T t$ satisfies $\mathscr{C}_{1}$. So we can replace $\alpha$ with $\forall \forall T t$. We can refine our choices of $\beta, \gamma$ similarly.

More definitions. For each $n \geq 1$, we define the relation $\operatorname{Neut}_{n}$ on terms $t, u$ of $L^{+}$, as follows:

$$
\begin{aligned}
\operatorname{Neut}_{1}(t, u) \Longleftrightarrow & \text { there is an assignment function } s \text { such that } \operatorname{Subf}\left(T t, \operatorname{Val}_{\mathscr{L}, s}(u)\right) \\
\operatorname{Neut}_{n}(t, u) \Longleftrightarrow & \text { there exist terms } r_{1}, \cdots, r_{n-1} \operatorname{such} \text { that }\left[\operatorname{Neut}_{1}\left(t, r_{1}\right)\right. \\
& \left.\& \operatorname{Neut}_{1}\left(r_{1}, r_{2}\right) \& \cdots \& \operatorname{Neut}_{1}\left(r_{n-2}, r_{n-1}\right) \& \operatorname{Neut}_{1}\left(r_{n-1}, u\right)\right] .
\end{aligned}
$$

We say that $t$ neutralizes $u$, written $\operatorname{Neut}(t, u)$, if there is some $n \geq 1$ such that Neut $_{n}(t, u)$. This terminology is motivated by Corollary 5 below. Note that Neut is the transitive closure of Neut 1 .

Lemma 5 Let $\varphi=\forall \forall$ Tt be a basic sentence, and suppose there are fixed points $f, h$ such that $f(\varphi)=\boldsymbol{n}$ but $h(\varphi) \neq \boldsymbol{n}$. Then there is a basic sentence $\psi=\forall \forall T w$ such that $f(\psi)=\boldsymbol{n}, h(\psi) \neq \boldsymbol{n}$, and $\mathrm{Neut}_{1}(w, t)$. 
Proof Since $f$ is a fixed point, $\operatorname{Val}_{\mathscr{L}+f}(\varphi)=f(\varphi)=\mathbf{n}$. By the Quantifier Property, there is an assignment function $s_{0}$ such that $\operatorname{Val}_{\mathscr{L}+f, s_{0}}(T t)=f\left(\operatorname{Val}_{\mathscr{L}+f, s_{0}}(t)\right)=\mathbf{n}$. Note that every term of $L^{+}$is a term of $L$, so $\operatorname{Val}_{\mathscr{L}+f, s_{0}}(t)=\operatorname{Val}_{\mathscr{L}+h, s_{0}}(t)$. Set $\theta=$ $\operatorname{Val}_{\mathscr{L}, s_{0}}(t)$. Note that $\theta \in \operatorname{Sent}\left(L^{+}\right)$, since fixed points assume the value $\mathbf{f}$ on $D-$ $\operatorname{Sent}\left(L^{+}\right)$.

Since $f$ is a fixed point, $\operatorname{Val}_{\mathscr{L}+f}(\boldsymbol{\theta})=f(\boldsymbol{\theta})=\mathbf{n}$. Also, since $h(\varphi) \neq \mathbf{n}$, we have that $\operatorname{Val}_{\mathscr{L}+h, s_{0}}(T t) \neq \mathbf{n}$, and hence $\operatorname{Val}_{\mathscr{L}+h}(\theta)=h(\theta) \neq \mathbf{n}$.

Applying Lemma 4 to $\theta$, we obtain a term $w$ satisfying (a) and (b). Then $\psi=$ $\forall \forall T w$ has all the desired properties.

Corollary 4 If $\varphi=\forall \forall T$ t is a basic sentence and there are fixed points $f, h$ such that $f(\varphi)=\boldsymbol{n}$ and $h(\varphi) \neq \boldsymbol{n}$, then there is a sequence of terms $\left(w_{i}\right)_{i \in \mathbb{N}}$ such that:

1. $w_{0}=t$,

2. $\operatorname{Neut}_{1}\left(w_{i+1}, w_{i}\right)$ for all $i \in \mathbb{N}$,

3. $f\left(\forall \forall T w_{i}\right)=\boldsymbol{n}$ and $h\left(\forall \forall T w_{i}\right) \neq \boldsymbol{n}$, for all $i \in \mathbb{N}$.

Lemma 6 Suppose that $\left(w_{i}\right)_{i \in \mathbb{N}}$ is a sequence of terms such that $\operatorname{Neut}_{1}\left(w_{i+1}, w_{i}\right)$ for all $i \in \mathbb{N}$. If $f$ is a fixed point of $\mu_{M}$, then the following function $g$ is also a fixed point:

$$
g(\varphi)= \begin{cases}n & \text { if there is a term } u \text { such that } \exists j \operatorname{Neut}\left(w_{j}, u\right) \text { and } \operatorname{Subf}(T u, \varphi) \\ f(\varphi) & \text { else. }\end{cases}
$$

Proof We must show that $\operatorname{Val}_{\mathscr{L}+g}(\varphi)=g(\varphi)$ for all sentences $\varphi \in \operatorname{Sent}\left(L^{+}\right)$. Divide into two cases, corresponding to the cases in the definition of $g$.

Case 1. Fix $j \in \mathbb{N}$, and suppose that there exists a term $u$ such that $\operatorname{Neut}\left(w_{j}, u\right)$ and $\operatorname{Subf}(T u, \varphi)$. We show that $\operatorname{Val}_{\mathscr{L}+g}(\varphi)=g(\varphi)=\mathbf{n}$.

Indeed, since $\operatorname{Neut}\left(w_{j+1}, w_{j}\right)$, there exists a term $r$ such that $\operatorname{Neut}\left(w_{j+1}, r\right)$ and $\operatorname{Neut}_{1}(r, u)$, i.e., there is an assignment function $s$ such that $\operatorname{Subf}\left(\operatorname{Tr}, \operatorname{Val}_{\mathscr{L}, s}(u)\right)$. It follows from the definition of $g$ that $g\left(\operatorname{Val}_{\mathscr{L}, s}(u)\right)=\mathbf{n}$. Now recall that $\operatorname{Subf}(T u, \varphi)$. Hence, by the key properties of the weak Kleene scheme, $\operatorname{Val}_{\mathscr{L}+g}(\varphi)=\operatorname{Val}_{\mathscr{L}+g, s}(\varphi)=$ $\mathbf{n}$, as desired.

Case 2. Suppose that $\varphi$ contains no subformula $T u$ such that $\exists j \operatorname{Neut}\left(w_{j}, u\right)$. Then $g(\varphi)=f(\varphi)$. We must show that $\operatorname{Val}_{\mathscr{L}+g}(\varphi)=g(\varphi)$. Since $f$ is a fixed point, it suffices to show that $\operatorname{Val}_{\mathscr{L}+g}(\varphi)=\operatorname{Val}_{\mathscr{L}+f}(\varphi)$. Hence, it suffices to check that, for any term $u$ and any assignment function $s$, if $\operatorname{Subf}(T u, \varphi)$, then $\operatorname{Val}_{\mathscr{L}+g, s}(T u)=$ $\operatorname{Val}_{\mathscr{L}+f, s}(T u)$, i.e., $g\left(\operatorname{Val}_{\mathscr{L}, s}(u)\right)=f\left(\operatorname{Val}_{\mathscr{L}, s}(u)\right)$.

Let $u$ and $s$ be arbitrary such that $\operatorname{Subf}(T u, \varphi)$. Let us calculate $g\left(\operatorname{Val}_{\mathscr{L}, s}(u)\right)$. Suppose for sake of contradiction that there are terms $w_{j}, r$ such that $\operatorname{Neut}\left(w_{j}, r\right)$ and $\operatorname{Subf}\left(\operatorname{Tr}, \operatorname{Val}_{\mathscr{L}, s}(u)\right)$. Then we have $\operatorname{Neut}\left(w_{j}, r\right)$ and $\operatorname{Neut}_{1}(r, u)$. Since Neut is transitive, it follows that Neut $\left(w_{j}, u\right)$, contrary to hypothesis. So, there is no such $r$.

Hence, by the definition of $g$, we have $g\left(\operatorname{Val}_{\mathscr{L}, s}(u)\right)=f\left(\operatorname{Val}_{\mathscr{L}, s}(u)\right)$ as desired.

Corollary 5 Suppose that $\operatorname{Neut}(t, u)$. If $h$ is any fixed point of $\mu_{M}$ and $h(\forall \forall T t)=\boldsymbol{n}$, then $h(\forall \forall T u)=\boldsymbol{n}$. 
Proof It suffices to prove this in the case where $\operatorname{Neut}_{1}(t, u)$. The proof is similar to case 1 of Lemma 6.

We now proceed to prove Theorem 2. By Corollary 3 , we can find basic sentences $\alpha, \beta, \gamma$ satisfying $\mathscr{C}$. Let $t_{\alpha}, t_{\beta}, t_{\gamma}$ denote the principal terms of $\alpha, \beta, \gamma$.

Divide into two cases, according to whether $g_{2}(\gamma)=\mathbf{v}_{\gamma}$ or $g_{2}(\gamma)=\mathbf{n}$.

Case 1: $g_{2}(\gamma)=v_{\gamma}$. In this case, we will construct a fixed point $g$ such that $g(\alpha, \beta, \gamma)=$ $\left(\mathbf{n}, \mathbf{n}, \mathbf{v}_{\gamma}\right)$. But then $g \notin\left\{g_{\min }, g_{1}, g_{2}, g_{3}, g_{\max }\right\}$, contradicting that the fixed-point poset of $\mu_{M}$ is isomorphic to $\mathscr{X}$.

Recall that $g_{2}(\alpha)=\mathbf{n}$, but $g_{\max }(\alpha) \neq \mathbf{n}$. Corollary 4 implies that there is a sequence of terms $\left(w_{i}\right)_{i \in \mathbb{N}}$ such that:

1. $w_{0}=t_{\alpha}$,

2. $\operatorname{Neut}_{1}\left(w_{i+1}, w_{i}\right)$ for all $i \in \mathbb{N}$, and

3. $g_{2}\left(\forall \forall T w_{i}\right)=\mathbf{n}$ and $g_{\max }\left(\forall \forall T w_{i}\right) \neq \mathbf{n}$, for all $i \in \mathbb{N}$.

Using this sequence $\left(w_{i}\right)$, we may apply Lemma 6 to $g_{\max }$ to obtain a fixed point $h$ such that:

$$
h(\varphi)= \begin{cases}\mathbf{n} & \text { if there is a term } u \text { such that } \exists j \operatorname{Neut}\left(w_{j}, u\right) \text { and } \operatorname{Subf}(T u, \varphi) \\ g_{\max }(\varphi) & \text { else. }\end{cases}
$$

Similarly, recall that $g_{3}(\beta)=\mathbf{n}$, but $g_{\max }(\beta) \neq \mathbf{n}$. By Corollary 4, we obtain a sequence of terms $\left(r_{i}\right)_{i \in \mathbb{N}}$ such that:

1. $r_{0}=t_{\beta}$,

2. $\operatorname{Neut}_{1}\left(r_{i+1}, r_{i}\right)$ for all $i \in \mathbb{N}$, and

3. $g_{3}\left(\forall \forall T r_{i}\right)=\mathbf{n}$ and $g_{\text {max }}\left(\forall \forall T r_{i}\right) \neq \mathbf{n}$, for all $i \in \mathbb{N}$.

Apply Lemma 6 again to $h$ to obtain a new fixed point $g$ such that:

$$
g(\varphi)= \begin{cases}\mathbf{n} & \text { if there is a term } u \text { such that } \exists k \operatorname{Neut}\left(r_{k}, u\right) \text { and } \operatorname{Subf}(T u, \varphi) \\ h(\varphi) & \text { else. }\end{cases}
$$

Since $g_{2}(\gamma) \neq \mathbf{n}$, it follows by Corollary 5 that no term of $\left(w_{i}\right)$ neutralizes $t_{\gamma}$. Moreover, since $g_{3}(\gamma) \neq \mathbf{n}$, it follows that no term of $\left(r_{i}\right)$ neutralizes $t_{\gamma}$. But since $\gamma$ is basic, the only term $u$ for which $\operatorname{Subf}(T u, \gamma)$ is $u=t_{\gamma}$. Hence $g(\gamma)=h(\gamma)=\mathbf{v}_{\gamma}$. So, this $g$ is a fixed point of $\mu_{M}$ in which $(\alpha, \beta, \gamma)=\left(\mathbf{n}, \mathbf{n}, \mathbf{v}_{\gamma}\right)$. Contradiction.

Case 2: $g_{2}(\gamma)=\boldsymbol{n}$. In this case, we will again derive a contradiction by showing that $\mu_{M}$ has too many fixed points. We will specify two more sentences $\delta, \varepsilon$, whose values will help us to show that the fixed points we construct are really distinct.

First, recall that $g_{3}(\beta)=\mathbf{n}$ but $g_{\max }(\beta) \neq \mathbf{n}$. By Corollary 4 , there is a sequence of terms $\left(u_{i}\right)_{i \in \mathbb{N}}$ such that:

1. $u_{0}=t_{\beta}$,

2. $\operatorname{Neut}_{1}\left(u_{i+1}, u_{i}\right)$ for all $i \in \mathbb{N}$, and

3. $g_{3}\left(\forall \forall T u_{i}\right)=\mathbf{n}$ and $g_{\max }\left(\forall \forall T u_{i}\right) \neq \mathbf{n}$, for all $i \in \mathbb{N}$. 
Recall that $g_{3}(\alpha) \neq \mathbf{n}$ and $g_{3}(\gamma) \neq \mathbf{n}$. By Corollary 5, no member of $\left(u_{i}\right)$ neutralizes $t_{\alpha}$ or $t_{\gamma}$.

Next, recall that $g_{1}(\gamma)=\mathbf{n}$ but $g_{\max }(\gamma) \neq \mathbf{n}$. By Corollary 4, there is a sequence of terms $\left(w_{i}\right)_{i \in \mathbb{N}}$ such that:

1. $w_{0}=t_{\gamma}$,

2. $\operatorname{Neut}_{1}\left(w_{i+1}, w_{i}\right)$ for all $i \in \mathbb{N}$, and

3. $g_{1}\left(\forall \forall T w_{i}\right)=\mathbf{n}$ and $g_{\max }\left(\forall \forall T w_{i}\right) \neq \mathbf{n}$, for all $i \in \mathbb{N}$.

Recall that $g_{1}(\alpha) \neq \mathbf{n}$. By Corollary 5 , no member of $\left(w_{i}\right)$ neutralizes $t_{\alpha}$.

Similarly, $g_{2}(\gamma)=\mathbf{n}$ but $g_{\max }(\gamma) \neq \mathbf{n}$. So, by Corollary 4 again, there is a sequence of terms $\left(r_{i}\right)_{i \in \mathbb{N}}$ such that:

1. $r_{0}=t_{\gamma}$,

2. $\operatorname{Neut}_{1}\left(r_{i+1}, r_{i}\right)$ for all $i \in \mathbb{N}$, and

3. $g_{2}\left(\forall \forall T r_{i}\right)=\mathbf{n}$ and $g_{\max }\left(\forall \forall T r_{i}\right) \neq \mathbf{n}$, for all $i \in \mathbb{N}$.

Recall also that $g_{2}(\beta) \neq \mathbf{n}$. Hence, by Corollary 5 , no member of $\left(r_{i}\right)$ neutralizes $t_{\beta}$.

We are almost done with preliminaries. Let us introduce one last piece of notation:

Notation. Let $g_{\left(w_{i}\right)}$ denote the fixed point obtained by applying Lemma 6 to $g_{\max }$ using the sequence $\left(w_{i}\right)$. Also, let us write $g_{\left(w_{i}\right)+\left(r_{i}\right)}$ to denote the fixed point obtained by applying Lemma 6 to $g_{\left(w_{i}\right)}$ using the sequence $\left(r_{i}\right)$. Explicitly:

$\begin{aligned} g_{\left(w_{i}\right)}(\varphi) & = \begin{cases}\mathbf{n} & \text { if there exists } u \text { such that } \exists j \operatorname{Neut}\left(w_{j}, u\right) \text { and } \operatorname{Subf}(T u, \varphi) \\ \mathbf{v}_{\varphi} & \text { else. }\end{cases} \\ g_{\left(w_{i}\right)+\left(r_{i}\right)}(\varphi) & = \begin{cases}\mathbf{n} & \text { if there exists } u \text { such that } \exists k \operatorname{Neut}\left(r_{k}, u\right) \text { and } \operatorname{Subf}(T u, \varphi) \\ g_{\left(w_{i}\right)}(\varphi) & \text { else. }\end{cases} \end{aligned}$

We may now finish the proof. By hypothesis, $\mu_{M}$ has no fixed point $h$ such that $h(\alpha, \beta, \gamma)=\left(\mathbf{v}_{\alpha}, \mathbf{v}_{\beta}, \mathbf{n}\right)$.

Consider the fixed point $g_{\left(w_{i}\right)}$. From the description of $\left(w_{i}\right)_{i \in \mathbb{N}}$, we have that $g_{\left(w_{i}\right)}(\gamma)=\mathbf{n}$ and $g_{\left(w_{i}\right)}(\alpha)=\mathbf{v}_{\alpha}$ (since no member of $\left(w_{i}\right)$ neutralizes $\left.t_{\alpha}\right)$. Hence, there exists $j \in \mathbb{N}$ such that $\operatorname{Neut}\left(w_{j}, t_{\beta}\right)$.

By the same reasoning, there exists $k \in \mathbb{N}$ such that $\operatorname{Neut}\left(r_{k}, t_{\alpha}\right)$.

Let $n=\max \{j, k\}$.

Let $\delta=\forall \forall T w_{n}$ and $\varepsilon=\forall \forall T r_{n}$. Note that $g_{\max }(\boldsymbol{\delta})=\mathbf{v}_{\delta} \neq \mathbf{n}$ and $g_{\max }(\varepsilon)=\mathbf{v}_{\varepsilon} \neq \mathbf{n}$.

Finally, we can show that $\mu_{M}$ has at least six distinct fixed points. Their distinctness is witnessed by the values of $\delta, \varepsilon, \beta$. Here they are:

$$
\begin{aligned}
g_{\max }(\delta, \varepsilon, \beta) & =\left(\mathbf{v}_{\delta}, \mathbf{v}_{\varepsilon}, \mathbf{v}_{\beta}\right) \\
g_{\left(u_{i}\right)}(\delta, \varepsilon, \beta) & =\left(\mathbf{v}_{\delta}, \mathbf{v}_{\varepsilon}, \mathbf{n}\right) \\
g_{\left(r_{i}\right)}(\delta, \varepsilon, \beta) & =\left(\mathbf{v}_{\delta}, \mathbf{n}, \mathbf{v}_{\beta}\right) \\
g_{\left(r_{i}\right)+\left(u_{i}\right)}(\delta, \varepsilon, \beta) & =\left(\mathbf{v}_{\delta}, \mathbf{n}, \mathbf{n}\right) \\
g_{\left(w_{i}\right)+\left(u_{i}\right)}(\delta, \varepsilon, \beta) & =\left(\mathbf{n}, \mathbf{v}_{\varepsilon}, \mathbf{n}\right) \\
g_{\min }(\delta, \varepsilon, \beta) & =(\mathbf{n}, \mathbf{n}, \mathbf{n}) .
\end{aligned}
$$

Contradiction. 
Acknowledgements My warmest thanks go to Anil Gupta, an excellent teacher and mentor, for his extensive comments on this manuscript. He caught my worst mistakes and clarified the argument considerably. I would also like to thank two anonymous referees for their very helpful comments.

\section{References}

1. Gupta, A., \& Belnap, N. (1993). The Revision Theory of Truth: MIT Press.

2. Kripke, S. (1975). Outline of a Theory of Truth. Journal of Philosophy, 72(19), 690-716.

3. Martin, R., \& Woodruff, P. (1975). On Representing 'True-in-L' in L. Philosophia, 5(3), 213-217.

4. Martínez-Fernández, J. (2007). Maximal Three-Valued Clones with the Gupta-Belnap Fixed-Point Property. Notre Dame Journal of Formal Logic, 48(4), 449-472.

5. Martínez-Fernández, J. (2014). Remarks on the Gupta-Belnap fixed-point property for $k$-valued clones. Journal of Applied Non-Classical Logics, 24(1-2), 118-131.

6. Visser, A. (2004). Semantics and the Liar Paradox. In Gabbay, D., \& Guethner, F. (Eds.) Handbook of Philosophical Logic, vol. 11, 2nd edn., (pp. 149-240): Springer. 